\title{
ESTUDIO DEL DESARROLLO DE LOS OSTRACODOS EUCYPRIS ARAGONICA Y HETEROCYPRZS SALINA EN CULTIVO DE BARRO
}

\author{
J. A. Marín \\ Departamento de Biología. Facultad de Ciencias. Universidad de Zaragoza.
}

Palabras Clave: Ostrocoda, development, mud cultures.

\author{
A B S TRACT \\ STUDY OF THE DEVELOPMENT OF THE OSTRACODA EUCYPRIS ARAGONICA AND \\ HETEROCYPRIS SALINA IN MUD CULTURE.
}

The development of the ostracoda Eucypris aragonica and Heterocypris salina was studied from mud cultures of the temporal saline lagoons "El Salobral" and "Rebollón" in the Bujaraloz-Sástago arid zone (Zaragoza), by means of their larval stages.

There is a high correlation between the length and the height of the valves in thedifferentstages. Thegrowth (length or heigth) related with the stage's number is potential type but its relationship with the time is of sigmoide type.

In both species, both sexes arose at the same time in the same proportion, but only H. salina has been fertile ( $37 \mathrm{eggs}$ per female as mean). The eggs hatched after the forth day.

Breaking out of appendages in the different larval stages of E. aragonica has also been studied.

\section{INTRODUCCION}

La expresión "cultivo de barro" se refiere a un procedimiento que permite desarrollarse a las formas resistentes que posiblemente se encuentren en el barro seco de una laguna temporal. Esta expresión equivale a las utilizadas anteriormente "cultivo de fango seco" (Margalef , 1953 p.152) y "cultures of imported dried rnud" (Hutchinson, 1975 p. 50).

Los cultivos de barro han sido aconsejados por varias razones:

-Dificultad de estudio de organismos acuáticos adaptados a periodos breves e irregulares de inundación con desarrollo rápido.

-Producción de formas resistentes a la desecación que quedan en el barro y que se desarrollan al volver a tener condiciones favorables.

Hay antecedentes de organismos descritos y

Limnética 1: 345-354 (1984)

(๑) Asociación Espaflola de Limnologia, Madrid. Spain estudiados, a veces accidentalmente, por medio de cultivos de barro como Branchinectella media y Eucypris aragonica (Margalef, 1953).

En este trabajo se estudia el crecimiento y desarrollo de E. aragonica y Heterocypris salina por medio de la observación de sus mudas.

\section{MATERIAL Y METODOS}

Cultivo de barro: El barro se recogió en la superficie del fondo seco de las lagunas temporales El Salobral y El Saladar (Bujaraloz), Pez y Rebollón (Sástago) en octubre de 1978 y se guardó en sobres de papel.

Los cultivos se desarrollaron en botes de vidrio destapados de unos $300 \mathrm{ml}$ de capacidad, cuyos fondos se cubrieron de barro seco y se llenaron de agua 
deionizada. Se colocaron a la luz de una ventana a la temperatura del laboratorio. Se iniciaron el 11-8-1980 y hacia el veinteavo día comenzaron a aparecer los individuos del cultivo de Rebollón iniciado el 31-3-81 nica no y los cultivos se repitieron estudiándose los individuos del cultio de Rebollón iniciado el 31-3-81 en el que aparecieron a los 8 días.

Un mes después de iniciado el cultivo se hicieron análisis químicos de los iones $\mathrm{Cl}^{-}$(valorado con $\mathrm{AgNO}_{3}$ ), $\mathrm{Ca}^{+2}$ y $\mathrm{Mg}^{+2}$ (valorados con EDT A) y se midió el pH.

Al aparecer los primeros individuos se colocaron separadamente en pocillos de vidrio de unos $4 \mathrm{~cm}$. de diámetro y $2 \mathrm{~cm}$ de altura con unos $10 \mathrm{ml}$. de agua del frasco de cultivo original y una pequeña cantidad de sustrato (barro y algas) y se cubrieron con un vidrio de reloj colocándolos a la luz de la misma ventana. Así, el 5-9-80 cuatro individuos de H. salina se colocaron en pocillos individuales desde el cultivo de la laguna El Salobral. Al día siguiente nació un nuevo individuo en uno de estos pocillos. Y el 9 y 10 de abril de 1981 se iniciaron seis pocillos con un individuo cada uno de E. aragonica desde el cultivo de la laguna de Rebollón, cinco de los cuales nacieron en el barro del primer pocillo.

Estudio de las mudas: Las mudas se recogieron bajo un estereomicroscopio con un pequeño tubo capilar colocándolas en una gota de glicerina sobre un portaobjetos. Se separaron las valvas y, puestas con la concavidad hacia abajo, se dibujó su imagen proyectada por un microscopio Visopan (Reicher) con objetivo de 10 aumentos. Se midió su longitud y sur altura con una escala fabricada dibujando la proyección de un micrómetro objetivo en las mismas condiciones. Los datos se procesaron en un microordenador Sinclair ZX Spectrum. El programa utilizado para el ajuste de curvas se basaba en la regresión lineal de los datos transformados. Las ecuadiones ajustadas fueron:

Recta $\quad y=a x+b$ Sigmoide $y=a /\left(1+b x^{n}\right)$ Exponencial $y=a e^{n x} \quad$ Sigmoide exp. $y=a /\left(1+b e^{n x}\right)$ Potencial $\quad y=a x^{n} \quad$ Hiperbólica $y=a x /(b+x)$

Potencial mod. $y=a x^{n}+b$.

La evaluación de la bondad del ajuste se realizó mediante un test $\mathrm{F}$ entre los valores observados y los calculados para cada ecuación. El máximo valor de $\mathrm{F}$ correspondió al mejor ajuste.

En el caso de E. aragonica se hicieron preparaciones de los apéndices de las mudas para el estudio de su aparición y desarrollo, montándolos en glicerina. Se han representado las figuras más demostrativas aunque no pertenezcan al mismo individuo.

\section{RESULTADOS}

Cultivos de barro: En los cultivos de barro de las lagunas aparecieron dos ostrácodos: Eucypris aragonica en los de Saladar, Pez y Rebollón y Heterocypris salina en los de Salobral. Además del anostráceo Branchinectella media y el rotífero Hexartbra fennica. También Ruppia maritima, Riella belicopbylla. Oscillatoria y Ulothrix.

Un mes despues de iniciados los cultivos se hicieron algunos análisis químicos del agua con los siguientes resultados:

$\begin{array}{lrc} & \text { Salobral } & \text { Rebollón } \\ \mathrm{pH}^{-} & 7.3 & 8.2 \\ \mathrm{Cl}^{-} & 81.2 & 146.4 \mathrm{meq} / 1 \\ \mathrm{Ca}^{++} & 22.8 & 38.9 \mathrm{meq} / 1 \\ \mathrm{Mg}^{++} & 88.6 & 141.3 \mathrm{meq} / 1\end{array}$

De los cinco individuos de $H$. salina estudiados, cuatro se recogieron en el tercer estadio y solo al nacido en el pocillo se le recogieron todas sus mudas, resultando ser en total tres machos y dos hembras.

De los seis individuos de E. aragonica, cinco se siguieron desde el primer estadio y uno desde el segundo. Resultando ser tres machos y tres hembras.

Estudio de las mudas: Los individuos recién nacidos mudaron ocho veces, siendo adultos en el noveno estadio. Las medidas de las valvas de sus mudas y la duración de los estadios están expresados en la tabla I para E. aragonica y e n la tabla III para $\mathbf{H}$. ralina.

l.as dimensiones de los individuos adultos oscilan entre los siguientes valores (se ha tomado siempre la media de las medidas de las valvas para cada individuo):

$\begin{array}{ccc}\text { E.aragonica } & \text { longitud } & \text { altura } \\ \text { machos } & 0.9-1.2 \mathrm{~mm} & 0.5-0.7 \mathrm{~mm} \\ \text { hembras } & 1.2-1.5 \mathrm{~mm} & 0.6-0.8 \mathrm{~mm} \\ & & \\ \text { H. salina } & \text { longitud } & \text { altura } \\ \text { machos } & 1.0-1.3 \mathrm{~mm} & 0.6-0.7 \mathrm{~mm} \\ \text { hembras } & 1.4-1.6 \mathrm{~mm} & 0.8-0.9 \mathrm{~mm}\end{array}$

En el cultivo de barro de la laguna de El Salobral había en un momento dado (29-10-80) nueve machos y treinta y dos hembras adultos. La aparición de sexos, sin embargo, fue al mismo tiempo y en una proporción parecida según los individuos 
Tabla I Longitud, altura y duración de los estadíos de E. aragonica.

Legnth, height and duration of the larval stages of E. aragonica.

INDIVIDUO 1

\begin{tabular}{|c|c|c|c|}
\hline estadio & longitu & altura & días \\
\hline 1 & $215 \mu \mathrm{m}$ & $150 \mathrm{um}$ & (1) \\
\hline 2 & 267.5 & 187.5 & 2 \\
\hline 3 & 312.5 & 210 & 2 \\
\hline 4 & 387.5 & 260 & 2 \\
\hline 5 & 480 & 317.5 & 3 \\
\hline 6 & 610 & 377.5 & 3 \\
\hline 7 & 752.5 & 445 & 6 \\
\hline 8 & 890 & 515 & 7 \\
\hline 9 & 1040 & 605 & 30 \\
\hline
\end{tabular}

INDIVIDUO 4

$\begin{array}{cllc}\text { estadio } & \text { longitud } & \text { altura } & \text { Días } \\ 1 & 210 & 150 & 1 \\ 2 & 277.5 & 185 & 2 \\ 3 & 110 & 217.5 & 5 \\ 4 & 390 & 2475 & 4 \\ 5 & 505 & 112.5 & 2 \\ 6 & 645 & 390 & 4 \\ 7 & 840 & 492.5 & 5 \\ 8 & 1155 & 650 & 5 \\ 9 & 1482.8 & 795 & 21\end{array}$

TABLA 11 Duración de vida de E. aragonaca Life duration of E. aragonica.
INDIVIDUO 2

\begin{tabular}{|c|c|c|c|}
\hline estadio & Longirud & Altura & Días \\
\hline 1 & $210 \mathrm{pm}$ & $150 \mu \mathrm{m}$ & 1 \\
\hline 2 & 262.5 & 190 & 1 \\
\hline 3 & 297.5 & 215 & 2 \\
\hline 4 & 365 & 252.5 & 3 \\
\hline 5 & 450 & 290 & 3 \\
\hline 6 & 537.5 & 1325 & 5 \\
\hline 7 & 660 & 3925 & 10 \\
\hline 8 & 790 & 445 & 7 \\
\hline 9 & 900 & 510 & 18 \\
\hline
\end{tabular}

INDIVIDUO 5

$\begin{array}{cccc}\text { estadio } & \text { longitud } & \text { altura } & \text { días } \\ 1 & - & - & 1 \\ 2 & 275 & 180 & 2 \\ 3 & 302.5 & 200 & 5 \\ 4 & 360 & 225 & 3 \\ 5 & 470 & 2875 & 3 \\ 6 & - & - & 3 \\ 7 & 797.5 & 465 & 3 \\ 8 & 1027.5 & 590 & 5 \\ 9 & 1190 & 682.5 & 25\end{array}$

INDIVIDUO 3

$\begin{array}{cllc}\text { estadio } & \text { longitud } & \text { altura } & \text { días } \\ 1 & - & - & (1) \\ 2 & 257.5 & 185 & (1) \\ 3 & 307.5 & 217.5 & 2 \\ 4 & 367.5 & 230 & 2 \\ 5 & 462.5 & 300 & 3 \\ 6 & 625 & 380 & 4 \\ 7 & 815 & 470 & 8 \\ 8 & 995 & 555 & 8 \\ 9 & 1170 & 640 & 22\end{array}$

INDIVIDUO 6

\begin{tabular}{cllc} 
estadio & \multicolumn{1}{c}{ longitud } & \multicolumn{1}{c}{ altura } & días \\
1 & - & - & 1 \\
2 & 282.5 & 195 & 2 \\
3 & 320 & 215 & 5 \\
4 & 382.5 & 237.5 & 4 \\
5 & 500 & 315 & 4 \\
6 & 660 & 395 & 3 \\
7 & 845 & 492.5 & 5 \\
8 & 1060 & 590 & 10 \\
9 & 1220 & 655 & 15
\end{tabular}

Length, heigth and duration of the arval stages of $\mathrm{H}$ salina.

INDIVIDUO 1

\begin{tabular}{cccc} 
estadio & longitud & \multicolumn{1}{c}{ altura } & días \\
1 & $\mu \mathrm{m}$ & $\mu \mathrm{m}$ & $(1)$ \\
2 & - & - & $(1)$ \\
3 & 275 & 180 & $(1)$ \\
4 & 362.5 & 230 & 1 \\
5 & 470 & 292.5 & 1 \\
6 & 625 & 370 & 1 \\
7 & 825 & 500 & 1 \\
8 & 1160 & 675 & 4 \\
9 & 1375 & 805 & 8
\end{tabular}

INDIVIDUO 2

$\begin{array}{cccc}\text { estadio } & \text { longitud } & \text { altura } & \text { días } \\ 1 & \mu \mathrm{m} & \mu \mathrm{m} & (1) \\ 2 & - & - & (1) \\ 3 & 307.5 & 192.5 & (1) \\ 4 & 400 & 245 & 1 \\ 5 & 490 & 287.5 & 1 \\ 6 & 657.5 & 375 & 1 \\ 7 & - & - & 1 \\ 8 & 1215 & 700 & 2 \\ 9 & 1580 & 925 & 31\end{array}$

INDIVIDUO 3

$\begin{array}{cccc}\text { estadio } & \text { longitud } & \text { altura } & \text { días } \\ 1 & 190 & 120 & (1) \\ 2 & 210 & 145 & 1 \\ 3 & 270 & 175 & 1 \\ 4 & 345 & 210 & 1 \\ 5 & 445 & 270 & 2 \\ 6 & 555 & 330 & 3 \\ 7 & 715 & 415 & 5 \\ 8 & 862.5 & 490 & 10 \\ 9 & 1000 & 560 & 11\end{array}$


INDIVIDUO 4

$\begin{array}{cccc}\text { estadio } & \text { longitud } & \text { altura } & \text { días } \\ 1 & - & - & (1) \\ 2 & - & - & (1 \\ 3 & 285 & 180 & (1) \\ 4 & 357.5 & 227.5 & 1 \\ 5 & 457.5 & 280 & 1 \\ 6 & 590 & 345 & 1 \\ 7 & 770 & 420 & 1 \\ 8 & 1000 & 550 & 3 \\ 9 & 1170 & 667.5 & 11\end{array}$

TABLA IV Duración de vida de H. salina. Life duration of $\mathrm{H}$. ralina
INDIVIDUO $\mathrm{S}$

$\begin{array}{cccc}\text { estadio } & \text { longitud } & \text { altura } & \text { días } \\ 1 & - & - & (1) \\ 2 & - & - & (1) \\ 3 & 285 & 182.5 & (1) \\ 4 & 365 & 220 & 1 \\ 5 & 465 & 275 & 1 \\ 6 & 615 & 345 & 1 \\ 7 & 802.5 & 435 & 1 \\ 8 & 1040 & 590 & 3 \\ 9 & 1270 & 710 & 19\end{array}$

\begin{tabular}{lcccccc} 
individuo & 1 & 2 & 3 & 4 & 5 & $\begin{array}{c}\text { media } \\
\text { (días) }\end{array}$ \\
\hline desarrollo & 11 & 9 & 24 & 10 & 1 & 12.8 \\
adulto & 8 & 31 & 11 & 11 & 19 & 16 \\
TOTAL & 19 & 40 & 35 & 21 & 29 & 28.8 \\
\hline
\end{tabular}

desarrollados en los pocillos.

Al llegar a adultos, o cuando ya estaban próximos a la última muda, se formaron varias parejas de macho y hembra entre los pocillos y entre los individuos del cultivo original y se colocaron en pocillos aislados. Su comportamiento fue distinto en las dos especies. En el caso de E. aragonica los intentos de cópula fueron pocos y en ningun caso hubo fertilidad (tampoco en los cultivos de barros originales).

Para H. salina las cópulas fueron frecuentes y las hembras comenzaron pronto a poner huevos, encontrándose espermatozoides en la disección de una hembra. Generalmente colocaron los huevos agrupados en montocillos y semienterrados, para lo cual utilizaban las antenas II y las patas II, además de piezas bucales

$\begin{array}{lllllll}\text { pareja } & 1 & 2 & 3 & 4 & 5 & 6\end{array}$

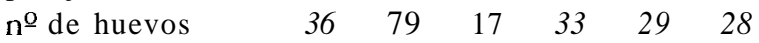

Estos huevos comenzaron a eclosionar rápidamente. Si los primeros huevos puestos fueron los primeros en nacer, los tiempos de aparición de los primeros individuos variaron entre 4-5 días a 13 días, según pocillo, frente a los 20 días que tardaron en el cultivo original, no necesitando ningún periodo de desecación para germinar. Los huevos despigmentados no eclosionaron.

En las tablas II y IV se resume la duracion del crecimiento y de la vida adulta de los individuos observados de E. aragonica y $H$. salina respectivamente. Se ha supuesto que en los primeros estadios no observados, la frecuencia de muda fue de 1 muda/día y se han sumado los días correspondientes a los registrados en las tablas 1 y 3 . La vida media de un individuo e n estas condiciones de cultivo fue de más de 7 semanas (51.6 días) para E. aragonica (extremos de 49 y 56 días) y de cerca de un mes (28.8 días) para $H$. salina que mostró gran variabilidad (entre 19 y 40 días).

Las dimensiones de las mudas (longitud y altura de las valvas) están muy correlacionadas en los distintos estadios. Para cada individuo los coeficientes de correlación $\mathrm{r}$ entre los logaritmos naturales de sus dimensiones fueron siempre mayores que 0.99 y sus representaciones resultan ser unas rectas muy ajustadas (figuras 1 y 2). Para cada individuo, los puntos que representan los estadios se van distanciando progresivamente entre sí, pero se uniformiza su distancia si se representan los logaritmos de sus dimensiones.

La representación de la longitud o de la altura frente al número de estadio es de tipo exponencial (figura 5a) y frente al tiempo es de tipo sigmoide (figura $5 b$ ).

El mejor ajuste entre las dimensiones y el número de estadio es la ecuación potencial modificada $\left(y=a x^{n}+b\right)$ en la que "b" se aproxima a la dimensión inicial del individuo. La ecuación exponencial $\left(y=a e^{n x}\right)$ también muestra un ajuste muy bueno. El valor de F a un nivel $\mathrm{P}=0.01$ fue siempre muy inferior a los obtenidos. 
El mejor ajuste entre las dimensiones y el tiempo corresponde a la ecuación sigmoide exponencial $\left(y=a /\left(1+b e^{n x}\right)\right.$ en la que "a", la asíntota, es próxima al valor final de la dimensión del individuo. Los puntos de inflexión de estas curvas corresponden al intervalo del 50 o $6^{0}$ estadio y más raramente a otros. En los pocos casos en que se ajusta mejor la ecuación potencial modificada, el valor de la asíntota "a" es mucho mayor al máximo valor de la dimensión y los puntos de inflexión caen fuera de la zona de crecimiento del individuo, correspondiendo el ajuste de los datos a la parte cóncava positiva de la sigmoide.

Del estudio de las preparaciones de las mudas de E. aragonica se ha establecido un orden de aparición de sus apéndices en los distintos estadios resumidos en el cuadro siguiente:

\begin{tabular}{|c|c|c|c|c|c|c|c|}
\hline estadio: & 1 & 23 & 3 & 5 & 6 & & 8 \\
\hline antenal & $\mathrm{x}$ & - & - & - & - & - & - \\
\hline antena II & $\mathrm{x}$ & - & . & - & - & - & - \\
\hline mandíbula & $\mathrm{r}$ & $\mathrm{x}$ & . & - & - & - & - \\
\hline maxila & & e & $\mathrm{x}$ & - & - & - & - \\
\hline pata I & & & & & $\mathrm{x}$ & & - \\
\hline pata II & & $r$ & 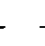 & $\mathrm{x}$ & - & - & - \\
\hline ata III & & & & & $\mathrm{e}$ & $\mathrm{x}$ & \\
\hline $\mathrm{rca}$ & & & & & $\mathrm{x}$ & - & \\
\hline
\end{tabular}

$\mathrm{e}=$ esbozo $\mathrm{r}=$ rudimento $\mathrm{x}=$ forma fina

Las figuras 3 y 4 representan los apéndices en los distintos estadios.

\section{DISCUSION}

Los cultivos de barro han sido una técnica idónea para el estudio del desarrollo de E. salina por su sencillez y por la adaptación de estos organismos a las condiciones de cultivo. Los demás animales aparecidos no se han adaptado tan favorablemente, muriendo probablemente debido a su mayor dependencia del medio planctónico.

La situación de los organismos y del medio ambiente en un cultivo de barro es diferente de la que se puede encontrar en las lagunas en condiciones naturales: en el cultivo, el agua permanece en condiciones más estables manteniendo un nivel constante y por lo tanto sin variaciones importantes de salinidad; la temperatura está amortiguada y alrededor de los 20-25ํㅜ $\mathrm{C}$; la composición química del agua, dependiente de las sales contenidas en el barro, difiere de los análisis efectuados en condiciones naturales. Además los organismos fueron puestos en un pequeño volumen de agua en los pocillos y con una pequeña cantidad de sustrato del cultivo original. El alimento podía ser escaso y la renovación del agua era muy pequeña o nula, acumulandose los detritos.

En estas condiciones se ha observado su desarrollo midiendo las mudas dejadas en los pocillos por los animales al crecer, por lo que no son medidas directas del animal vivo.

A pesar de ésto, la información recogida tiene la utilidad de mostrar el desarrollo de organismos adaptados a condiciones irregulares y de dificil estudio.

El ajuste de los datos de las dimensiones frente al número de estadio ha sido significativo en varias ecuaciones, pero especialmente bueno en la ecuación potencial modificada $\left(y=a x^{n}+b\right) y$, algo menos, en la ecuación exponencial $\left(\mathrm{y}=\mathrm{a} \mathrm{e}^{\mathrm{nx}}\right)$ La ecuación exponencial tiene el atractivo de que el cociente entre los valores para dos estadios consecutivos es igual a una constante y que los valores de las dimensiones en cada estacio serían los términos de una progresión geométrica de razón e.

En la ecuación potencial modificada el cociente entre los valores ( $y$-b) de dos estadios consecutivos no es una constante, sino que decrece al aumentar el estadio tomando los valores $(2 / 1)^{\mathrm{n}},(3 / 2)^{\mathrm{n}}, \ldots$, $(9 / 8)^{n}$ El crecimiento de estos individuos se ajusta más a curvas de la forma $x^{n}$, con $n$ positivo, pero $n$ entero, que a curvas de la forma $\mathbf{e}^{\mathrm{nx}}$, pero en los dos casos se obtiene un buen ajuste. Esto puede indicar la existencia de una relación alométrica entre las dimensiones y el número de estadio, considerándolo como un parámetro biológico.

El ajuste de las dimensiones y el tiempo a la ecuación sigmoide exponencial $\left(y=a /\left(1+b e^{n \times}\right)\right.$, altamente significativo, indica que el crecimiento se ve frenado por un factor limitante: al avanzar el tiempo el crecimiento se hace menor. Solo no se cumple en algún individuo de E. aragonica (el 4 y parcialmente el 5) en que una ecuación potencial modificada da un ajuste mejor, sin presencia de factor limitante.

El factor o conjunto de factores que limitan el crecimiento podrían estar relacionados con las características del cultivo y de los pocillos, pero influvendo más en retrasar el día de muda que en disminuir el tamaño del individuo.

El hecho de que en $H$. salina el crecimiento sea más rápido que en E. aragonica puede estar relacionado con su fertilidad en estos cultivos. E. 
350

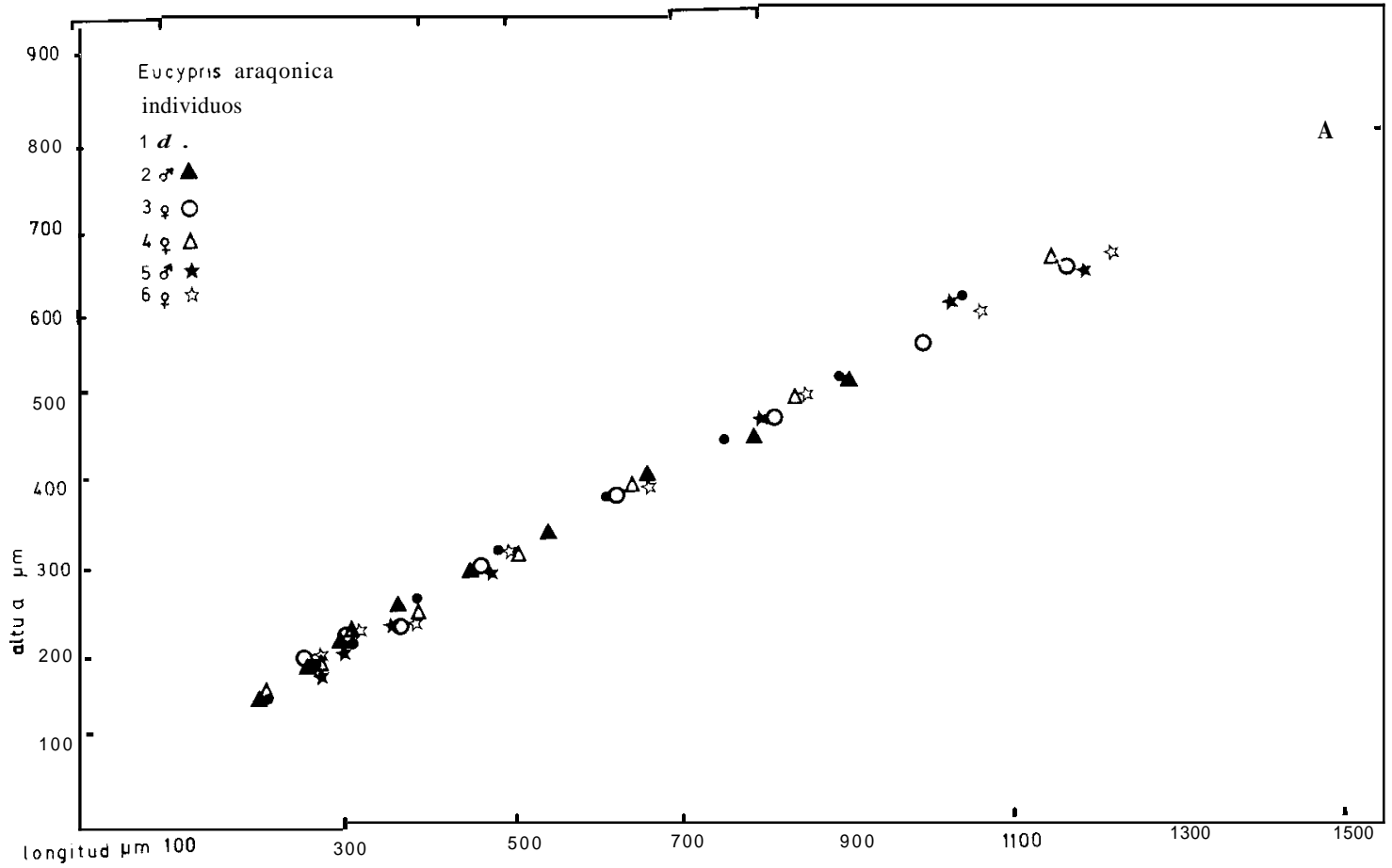

Figural 1 - Longitude y altura de 1 a a mazuma en los distintos estadios

Length and height of the valves of $l:$ arazmention different larval stages

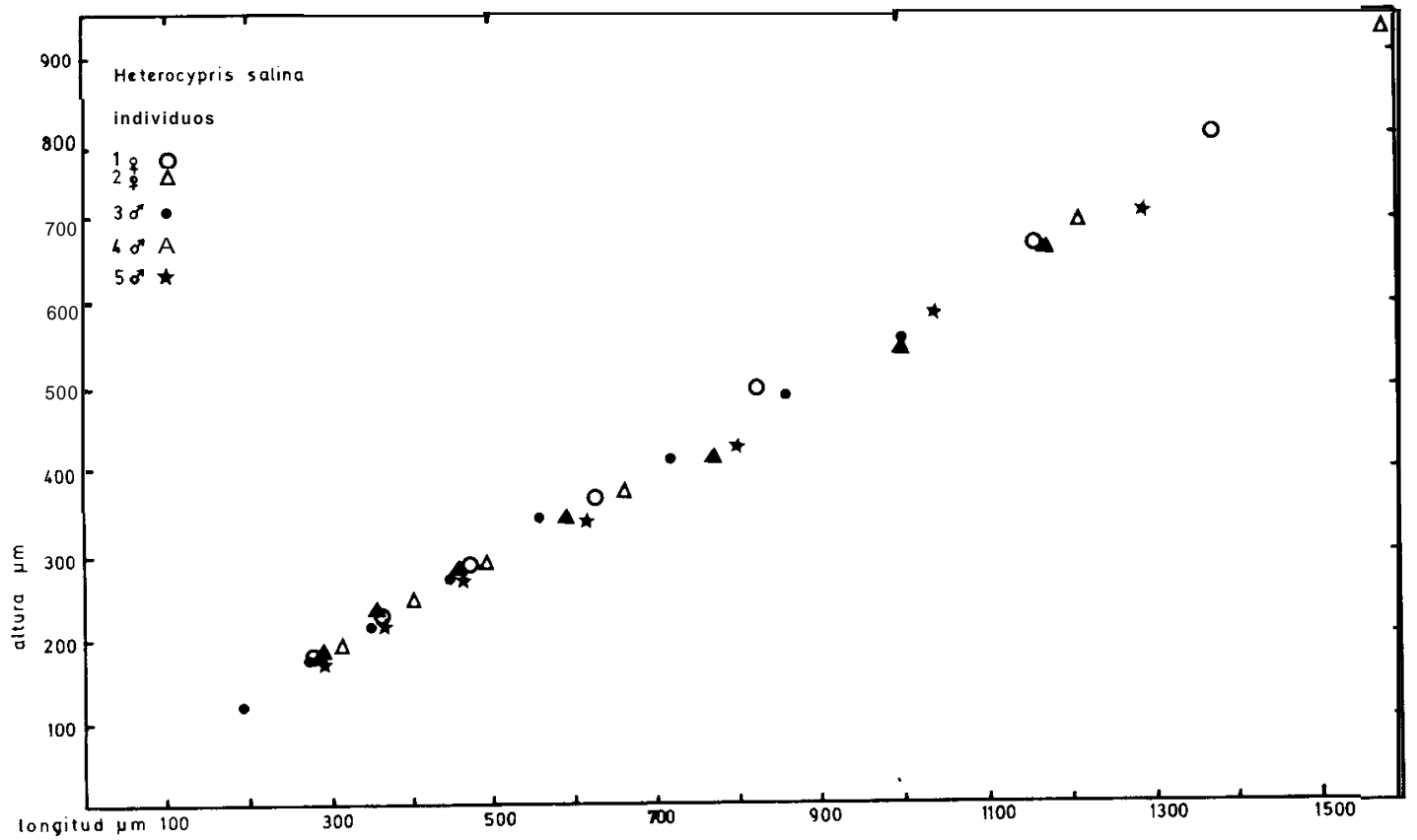

Figura 2.- Longitude y altura de las valves de H. salina en los distintos estadios Length and heigth of the valves of $\mathrm{H}$. salina in the different larval stages. 


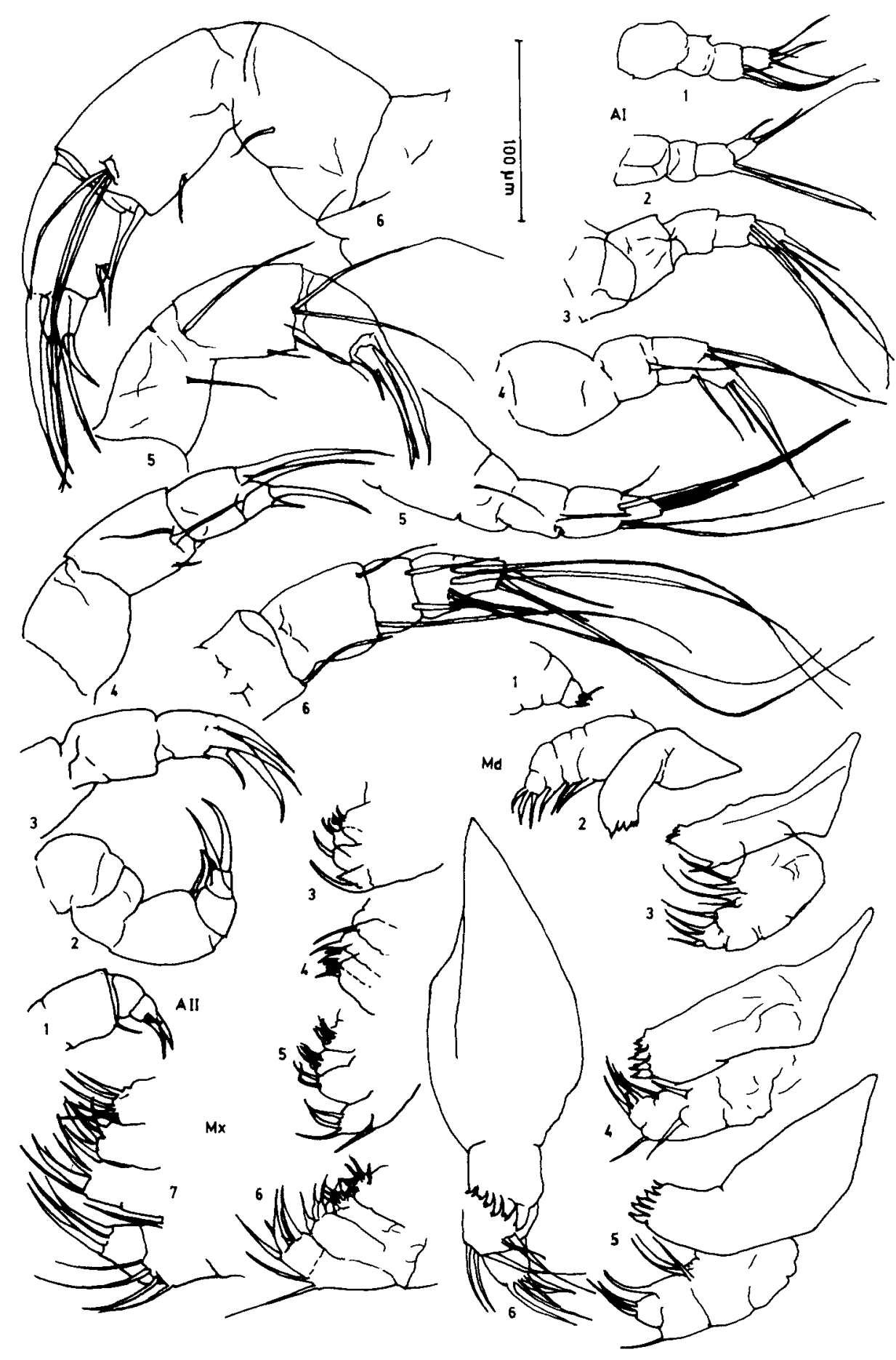

Figura 3 - Apendices de E. aragontca en los distintos estadíos Appendages of E. aragonica in the different larval stages. 


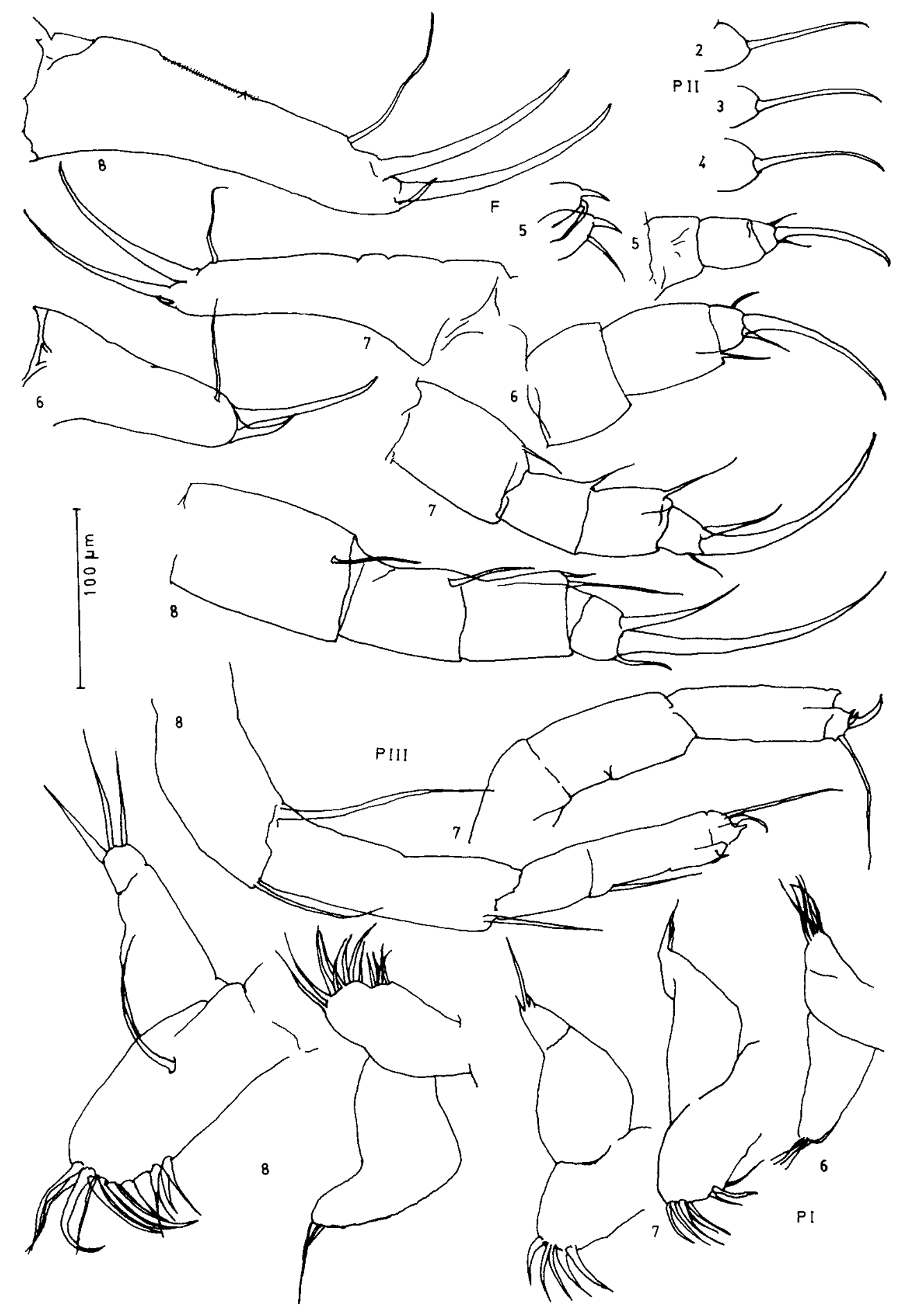

Figura 3 - (Continuación 


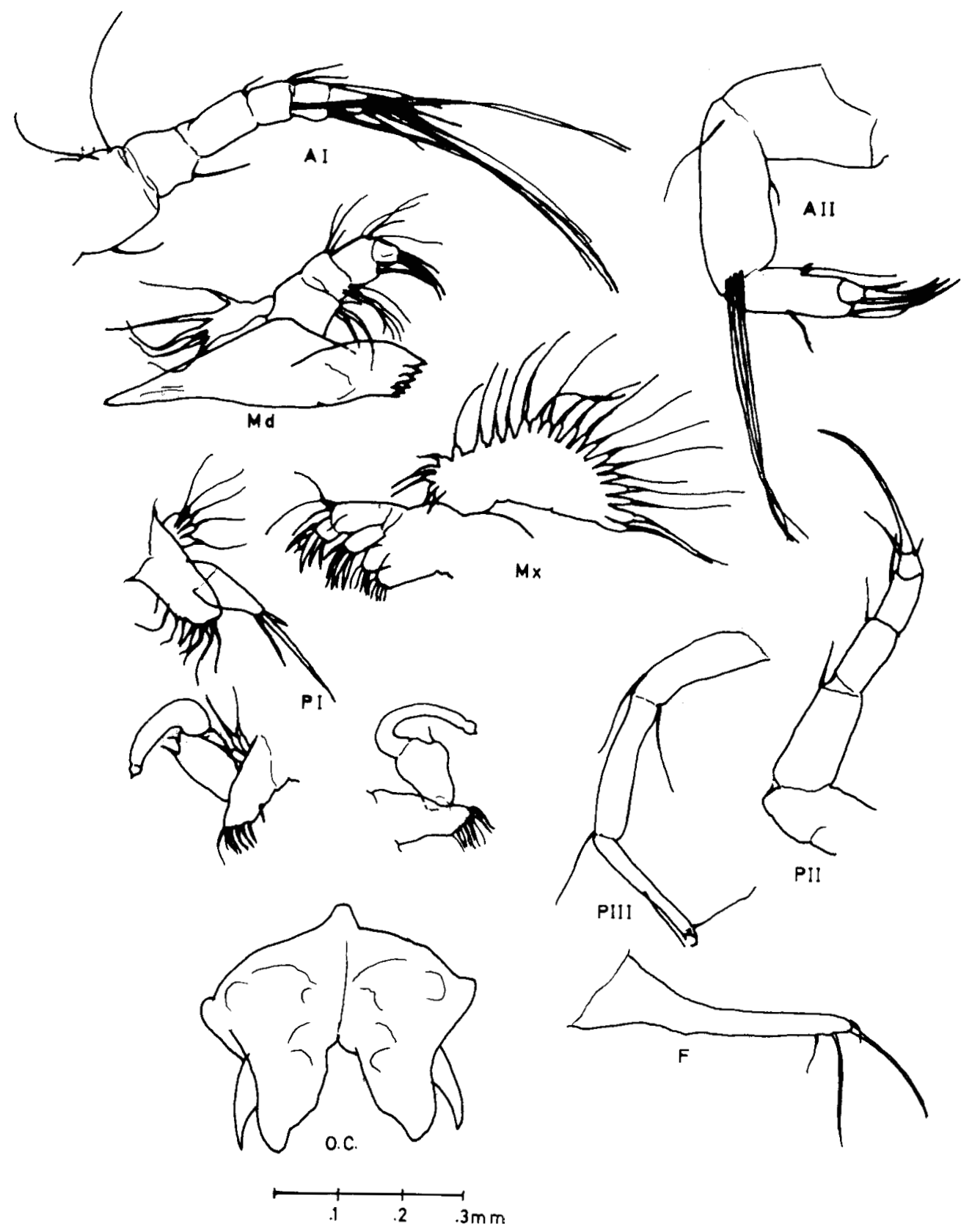

Figura 4.- Apéndices de E. aragonica en el estadio adulto

Appendinges of $1:$ aliagentiat in the adult stages 


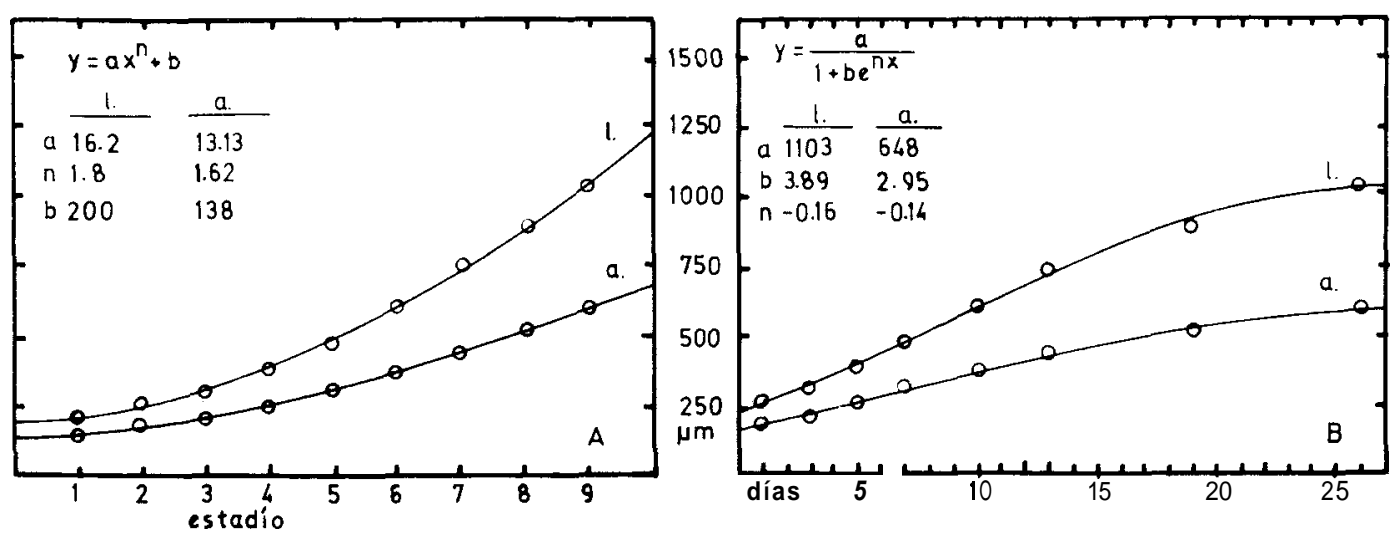

Figura 5.- Relación entre las dimensiones (longitud (I) y altura (a) y el númerodeestadio (A)oel tiempo (B) en E. aragonıca (individuo l) Relationship between dimension (length (1) and height (a) and stage number (A) or time (B) in E. aragontca (individual 1).

aragonica no ha sido fértil en los pocillos ni en los cultivos de barro en ninguna de las repeticiones hechas. Es probable que algún estímulo que encuentre en la naturaleza, como el aumento progresivo de la salinidad, las concentraciones elevadas de algún ión, la desecación,..., sea necesaria para desencadenar la reproducción.

Los huevos de H. salina no necesitaron ningún periodo de desecación para germinar, por lo que se han podido conservar individuos en cultivo durante mucho tiempo (más de un año, hasta que se dejó secar el cultivo).

Los sexos aparecen al mismo tiempo en las dos especies y en proporciones similares. Los cambios que experimenta esta proporción con el tiempo podría ser debido a una mayor mortalidad de los machos.

La descripción de la aparición y desarrollo de los apéndices en los estadios es a grandes rasgos similar en Claus, 1878, y en Hartmann, 1966, pero hay pequeñas diferencias en algún caso y diferentes interpretaciones a la naturaleza del apéndice aparecido en el estadio segundo. Para Hartmann se trata de la furca y para Claus de la pata del segundo par. En nuestro caso dada la similitud de la espina del último apéndice de los estadios 2,3 y 4 con la espina de la segunda pata ya formada (a partir del quinto estadio) y las diferencias con la furca que aparece en el quinto estadio con dos espinas más cortas, es mejor pensar que se trata del segundo par de patas con la forma rudimentaria.

\section{BIBLIOGRAFIA}

Claus, C. 1878.- Traité de Zoologie (Trad. $3^{\text {i }}$ ed. alemana) Librairie F. Savy Paris.

Hartmann, G. 1966. "Ostracoda". En Klassen und Orderungen des Tierreichs. (Bronns, H.G. ed.) 2 Buch, IV Teil Akademische Verlagsgeselischaft Gees \& Portig K.G., Leipzig.

Hutchinson, G.E. 1975.- A Treatise on Limnology. Limnological Botany Vol III John Wiley \& Sons. New York.

Margalef, R. 1953.- Los crustáceos de las aguas continentales ibéricas Instituto Forestal de Investigaciones y Experiencias Ministerio de Agricultura Madrid 